\title{
Analysis of Tropospheric Scintillation Intensity on Earth to Space in Malaysia
}

\author{
${ }^{1}$ Mandeep Singh Jit Singh, ${ }^{1}$ Syed Idris Syed Hassan, ${ }^{1}$ Mohd Fadzil Ain \\ ${ }^{2}$ Kiyoshi Igarashi, ${ }^{2}$ Kenji Tanaka and ${ }^{3}$ Mitsuyoshi Iida \\ ${ }^{1}$ School of Electrical and Electronic Engineering, University Sains Malaysia, Engineering \\ Campus, 14300,Nibong Tebal, Seberang Perai Selatan, Pulau Pinang. Malaysia \\ ${ }^{2}$ National Institute of Information and Communications Technology, Nukui-kita, Koganei, Tokyo , Japan \\ ${ }^{3}$ Association of Radio Industries and Businesses, Kasumigaseki, Chiyoda, Tokyo, Japan
}

\begin{abstract}
This paper presents the results of a study on tropospheric scintillation on satellite link that has been performed at University Sains Malaysia (USM) to obtain statistics of scintillation from the 12.255 GHz Superbird C satellite with an elevation angle of $40.1^{0}$. The objective of this analysis was to determine whether the long-term scintillation follows the log normal or gamma distribution. It was found that the long term probability distribution follows the log-normal distribution. The short term probability distribution also follows the log-normal model for the driest and the wettest month. It has been proven that the log-normal model is also suitable for prediction at moderate angles.
\end{abstract}

Keywords: Meteorology, propagation, satellite communication

\section{INTRODUCTION}

Rain induced attenuation on satellite earth link at frequencies above $10 \mathrm{GHz}$ is the predominant signal impairment. However, for design of low margin systems, especially those at high frequency and low elevation angles, scintillation effects must be accounted for to complete a link budget accurately ${ }^{[1]}$.

Tropospheric scintillation is a rapid fluctuation of signal amplitude and phase due to turbulent irregularities in temperature, humidity and pressure, which translate into small-scale variations in refractive index. In the microwave region, where the humidity fluctuation are important, the result is random degradation and enhancement in signal amplitude and phase received on a satellite link, as well as a degradation in performance of large antennas ${ }^{[2]}$.

Description of Sintillation Effects: Tropospheric scintillation is characterized by two main parameters ${ }^{[3]}$. Firstly, the log-amplitude $\chi$ (in decibels) which is, the ratio of the instantaneous amplitude of the observed signal to the mean amplitude, expressed in decibels (dB). The mean amplitude is generally calculated by a 60-s moving average filter (cutoff frequency around $0.025 \mathrm{~Hz})^{[3]}$ in order to separate rapid turbulence induced scintillation from spacecraft-induced variations and contributions of other propagation factors Secondly, the scintillation variance, $\sigma^{2}$ in $\mathrm{dB}^{2}$; that is, the variance of the log-amplitude $\chi$. In order to produce a good estimator of the instantaneous scintillation effect and accounting for its time-variability, the variance is generally calculated in sliding way over about one minute $^{[3]}$.

Scintillation is essentially a non-absorptive effect, which means that the mean level of the signal doesn't change immediately. It appears that a scintillation event is characterized by a stationary period of few minutes (up to ten or fifteen minutes). Within this period, the scintillation intensity remains constant and the scintillation log-amplitude distribution can be fitted quite well by a zero-mean Gaussian distribution, whose scintillation intensity is the mean scintillation intensity during the period. For a period longer than several minutes, the variability of $\sigma$, must be taken into account and the long-term probability density function (PDF) of the scintillation amplitude can be modeled by a Gaussian distribution with lognormally distributed scintillation intensity ${ }^{[3]}$.The cumulative distribution $\mathrm{P}\left(\chi>\chi_{0}\right)$ of $\chi$ can be written as.

$\mathrm{P}\left(\chi>\chi_{0}\right)=\int_{\chi_{0}{ }^{\circ}}^{\infty} p\left(\chi \mid \sigma_{\chi}\right) \cdot p\left(\sigma_{\chi}\right) d \sigma_{\chi} d \chi$

The short term PDF of $\chi$ follows a Gaussian distribution is written as

Corresponding Author: $\quad$ Mandeep Singh Jit Singh, ${ }^{1}$ School of Electrical and Electronic Engineering, University Sains Malaysia, Engineering Campus, 14300,Nibong Tebal, Seberang Perai Selatan, Pulau Pinang Malaysia 
$p\left(\chi \mid \sigma_{\chi}\right)=1 /\left(\sqrt{2 \pi \sigma_{\chi}}\right) \exp \left(-\chi /\left(2 \sigma_{\chi}^{2}\right)\right.$

The long term PDF which is log-normally distributed is given by

$p\left(\sigma_{\chi}\right)=1 /\left(\sqrt{2 \pi} \sigma_{\chi} s\right) \exp \left\{-\left[\ln \left(\sigma_{\chi}^{2} / \mathrm{m}\right)\right]^{2} /\left(2 s^{2}\right)\right\}$

where $\chi_{0}$ is a given threshold value, $m$ is the median value of scintillation intensity distribution, $\sigma_{\chi}$ in $\mathrm{dB}$ and $s$ is standard deviation of $\ln \left(\sigma_{\chi}^{2}\right)$

Most of the statistical models usually try to link the parameters of this PDF to ground meteorological measurements, such as temperature, relative humidity and wind velocity.

Experiment and Data Analysis: Starting from January 2002 until December 2002, the Superbird C satellite beacon measurement were done at USM, Malaysia at an elevation angle of $40.1^{\circ}$ and a sampling rate of $1 \mathrm{~Hz}$, using an offset Cassegrain antenna of diameter $2.4 \mathrm{~m}$.

The scintillation amplitude calculation was done during clear sky (absent of rain and spurious spikes) Spurious and invalid data have been eliminated by visual inspection of all data sequences before using the measured raw beacon propagation data for scintillation studies

The raw data was transformed from quantization levels to relative signal level, $\mathrm{A}$ in $\mathrm{dB}$. The power spectral density (psd), $\mathrm{dB}^{2} / \mathrm{Hz}$, of each signal was computed on a block of 4096 samples by breaking the block into seven half-overlapping segments of 1024 samples, removing the mean from each segment before multiplying by a Hanning window and finally averaging the periodograms (square magnitude FFT) of the modified segments. The psd was then smoothed using a third-order median filtering ${ }^{[4]}$.

Next the scintillation amplitude, $\chi \mathrm{dB}$ was extracted from $\mathrm{A}$ by passing the data through a Butterworth high-pass filter of fifth order. The filter was intended to remove the contribution of low frequency components from the signal, generally associates with rainfall fades or due to satellite motion. To examine the effect on scintillation statistics of the cutoff frequency $f_{\mathrm{c}}$, used for data filtering, of different values of $f_{\mathrm{c}}$ between 2 to $500 \mathrm{mHz}$ were used. For each scintillation data set (obtained using a particular value of $f_{\mathrm{c}}$ ) a goodness-of-fit test were performed to determine whether the 1-minute distribution of the scintillation amplitude followed a Gaussian $\mathrm{PDF}^{[4]}$.

The 1-minute scintillation amplitude was then averaged over 1-hour periods and the mean hourly intensities for each particular month were estimated.

The PDF of amplitude scintillation were then computed by dividing the histogram of the scintillation amplitude for certain period of time by the sum of the histogram multiplied by the bin size. Histogram of amplitude scintillation was computed by grouping them according to their amplitude in bins of $0.05 \mathrm{~dB}$.

Probability Density Function: Two model are generally accepted for the standard deviation of the signal amplitude, the Karasawa Gamma ${ }^{[5]}$ distribution and the Moulsley-Vilar lognormal distribution ${ }^{[6]}$. The Karasawa gamma distribution describes the distribution of signal standard deviation using parameters $\beta$ and $\zeta$ that could be related to measurements.

$f=\underline{\beta^{\zeta} \cdot \sigma_{x}} \frac{(\zeta-1) \cdot \exp \left(-\beta \cdot \sigma_{x}\right)}{\Gamma(\zeta)}$

where,

$$
\beta=\mathrm{m} / \sigma_{x}^{2} \quad \zeta=\mathrm{m}^{2} / \sigma_{x}^{2}
$$

The probability density for the log-normal distribution is given by,

$f=1 /\left(\sigma_{\sigma} \cdot \sigma_{\mathrm{m}}\right) \cdot \sqrt{2 / \pi} \exp \left\{-\left[\ln \left(\sigma_{\mathrm{x}}{ }^{2} / \sigma_{\mathrm{m}}{ }^{2}\right)\right]^{2} /\left(2 \sigma_{\sigma}^{2}\right)\right\}$

where, $\mathrm{m}=\ln \left(\sigma_{\mathrm{m}}{ }^{2}\right)$ and $\sigma_{\sigma}=\operatorname{var}\left(\ln \left(\sigma_{\mathrm{x}}^{2}\right)\right.$

\section{RESULTS AND ANALYSIS}

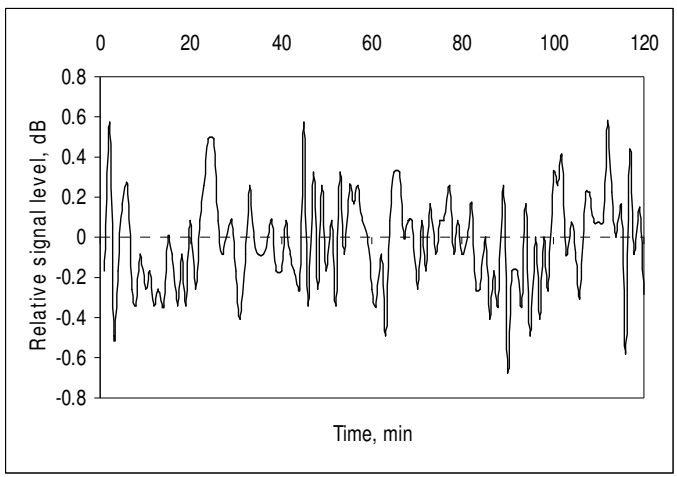

Fig. 1: Example of scintillation observed from beacon on a clear day. 


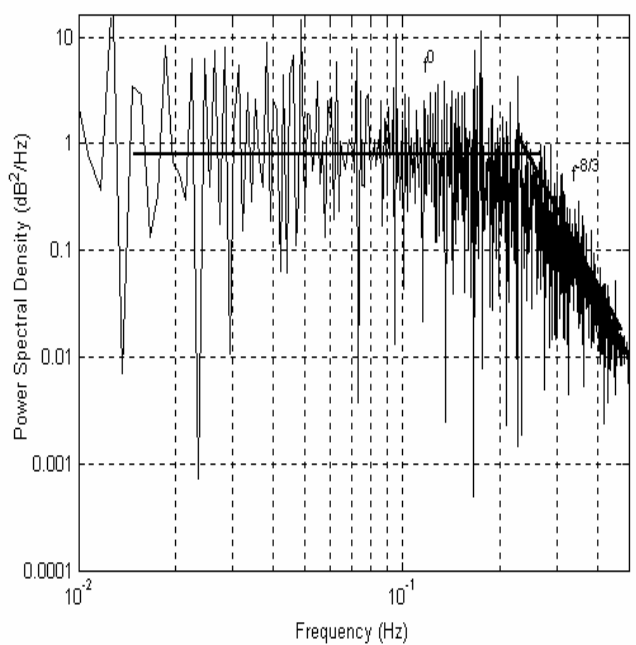

Fig. 2: Comparison of the measured scintillation spectrum with the theoretical pattern (straight line)

Figure 1 shows an example of the signal level variation caused by scintillation on a clear day with the present of cumulus clouds. The $0 \mathrm{~dB}$ level on the vertical axis is set to the clear sky. The field scintillation on space earth paths appears to be generally enhanced when clouds are present. The cloud liquid water, whose irregular spatial distribution contributes to an increase in the fluctuations of refractivity and in turn the scintillation, is also responsible for coexisting attenuation.

Figure 2 shows the power spectra of the scintillation presented in Fig. 1. The shapes of the power spectra is similar by the one recommended by Tatarski ${ }^{[7]}$, where the scintillation spectrum has a well known pattern of lowpass filtered white noise with a $-8 / 3$ slope, for fully developed turbulence. There is a really good agreement found between the measured scintillation spectrum and the theoretical spectrum (straight solid line), considering as usual in slant path that scintillation is produced by a thin (cloudy) layer of well developed tropospheric turbulence at a height between $1000 \mathrm{~m}$ and $3000 \mathrm{~m}$

The spectrum corner frequency $f_{c}$, at which the spectral density begins to fall, is related to the dynamic characteristics of scintillation, which depends on the transverse wind speed, $\mathrm{v}_{\mathrm{t}}$ the wave number, $\mathrm{k}$ and the slant path length, $\mathrm{L}$ through the turbulent layer. In the case of Fig. 2, $f_{c}$ is equal to $0.25 \mathrm{~Hz}$ and the flat region is from $0.02 \mathrm{~Hz}$ to $0.25 \mathrm{~Hz}$.

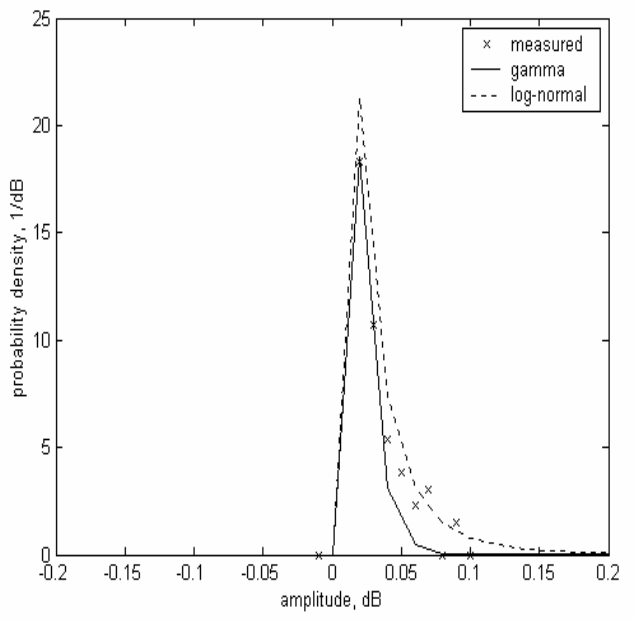

Fig.3: Short-term probability distribution for the driest month.

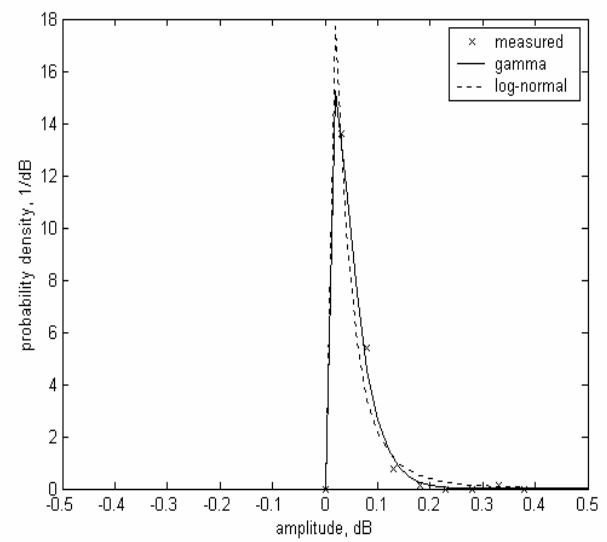

Fig. 4: Short-term probability distribution for the wettest month

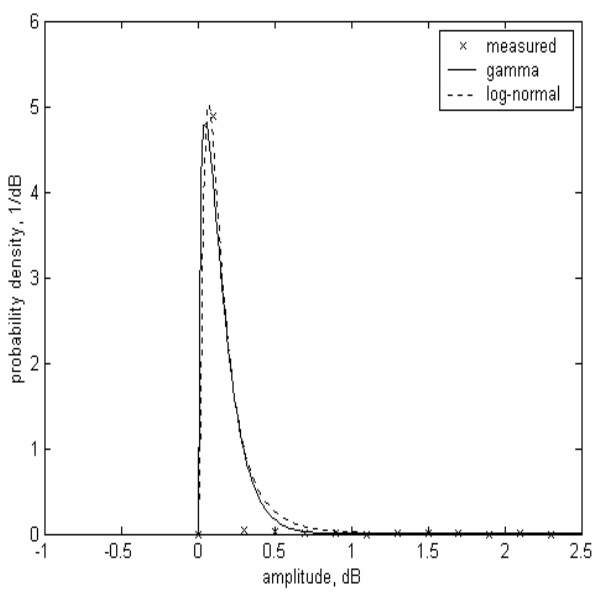

Fig. 5: Long -term probability distribution 
Based on Fig. 3, 4 and 5 its shows, that the Gamma distribution follows the short term distribution whereas the log-normal PDF follows the long term distribution. As suggested by Ortigies ${ }^{[8]}$ for higher elevation angle path, it appears that the log-normal distribution is more adequate for fitting the measured PDF, especially for scintillation intensity below $2 \mathrm{~dB}$. The strength of the scintillation is minimum for the driest month of $0.05 \mathrm{~dB}$ and maximum for the wettest month of $0.065 \mathrm{~dB}$

\section{CONCLUSION}

The tropospheric scintillation propagation experiment offers a good opportunity to investigate the scintillation intensity on moderate elevation angle earth-space path at millimeter wavelength. Not only for low elevation angle, but for moderate elevation angle the log-normal PDF follows the long-term probability distribution.

\section{ACKNOWLEDGMENT}

The authors would like to thank University Sains Malaysia and POST-PARTNERS Council for the equipment and support in undertaking this research.

\section{REFERENCES}

1. C.E. Mayer, B.E., Jaeger, and R.K. Crane.1997 Ka-Band Scintillations: Measurements and Model Predictions..IEEE Proc., vol.85,pp. 936-945

2. M.J.L, Kamp, ,J.K. Tervonen, E.T. Salonen, , P.V. Poiares. 1999 Improved Models for Long-Term Prediction of Tropospheric Scintillation on Slant Paths. IEEE Trans. Antenn. Propagat. Vol.47.pp. 249-260
3. H. Vasseur, 1999 Prediction of Tropospheric Scintillation on Satellite Links from Radiosonde Data. IEEE Trans. On Antenna\&Prop.,vol.47, pp.293-300

4. I.E. Otung, M.O. Al-Nuaimi and B.G. Evans. 1998.Extracting Scintillation From Satellite Beacon Propagation data. IEEE Trans. On Antenna\&Prop.,vol.48, pp.1580-1581

5. Y, Karasawa, M. Yamada and K, Yakusawa 1988. Tropospheric Scintillation in the 14/11$\mathrm{GHz}$ Bands on Earth Space Paths with Low Elevation Angles. IEEE Trans. Antenn. Propagat. vol.36, pp.563-569

6. T, Mousley and E, Vilar. 1982. Experimental and Theoretical Statistics of Microwave Amplitude Scintillation". Electronic Lett. Vol.31, pp. 1328-1329

7. V.I. Tatarksi. 1961 Wave Propagation in a Turbulent Medium. New York: Dover

8. G. Ortgies.1993.Prediction of Slant path Amplitude Scintillation from Meteorological Parameters. Proc.Int.Symp. on Radio Propogation, Beijing, pp.218-221. 